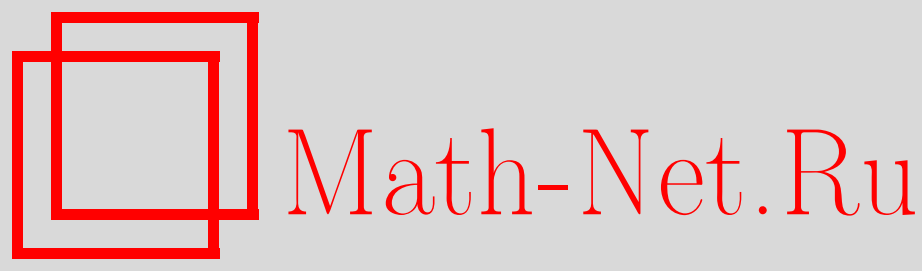

А. Л. Катаев, С. В. Михайлов, Новое представление эффектов нарушения конформной симметрии в калибровочных теориях поля в теории возмущений, ТМФ, 2012, том 170, номер 2, 174-187

DOI: https://doi.org/10.4213/tmf6757

Использование Общероссийского математического портала Math-Net.Ru подразумевает, что вы прочитали и согласны с пользовательским соглашением http://www . mathnet.ru/rus/agreement

Параметры загрузки:

IP : 34.229 .45 .116

26 апреля 2023 г., 15:06:53

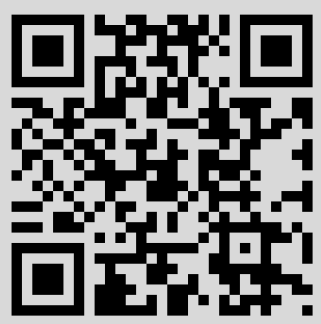




\title{
НОВОЕ ПРЕДСТАВЛЕНИЕ ЭФФЕКТОВ НАРУШЕНИЯ КОНФОРМНОЙ СИММЕТРИИ В КАЛИБРОВОЧНЫХ ТЕОРИЯХ ПОЛЯ В ТЕОРИИ ВОЗМУЩЕНИЙ
}

\begin{abstract}
Предложена гипотеза о детальной структуре представления для нарушающего конформную симметрию члена в обобщенном в рамках теории возмущений основном соотношении Крютера в случае КХД, перенормированной в схеме $\overline{\mathrm{MS}}$. Установлена справедливость данного представления в приближении $O\left(\alpha_{\mathrm{s}}^{4}\right)$. Использование сформулированного в работе варианта обобщенного соотношения Крютера позволяет найти связи между конкретными вкладами в коэффициенты ряда теории возмущений КХД для несинглетной по аромату части функции Адлера электрон-позитронной аннигиляции в адроны $D_{\mathrm{A}}^{\mathrm{ns}}$ и в коэффициенты ряда теории возмущений для правила сумм Бьеркена $S_{\mathrm{Bjp}}$ глубоконеупругого рассеяния поляризованных лептонов на нуклонах. Найдены новые соотношения для коэффициентов разложения при $\alpha_{\mathrm{s}}^{4}$ для $D_{\mathrm{A}}^{\mathrm{ns}}$ и $S_{\mathrm{Bjp}}$. Выполнение одного из них служит дополнительной теоретической проверкой недавних компьютерных аналитических расчетов членов порядка $\alpha_{\mathrm{s}}^{4}$ в выражениях для этих двух физических величин.
\end{abstract}

Ключевые слова: квантовая теория поля, нарушение конформной симметрии, ряды теории возмущений, ренормгруппа, связь характеристик инклюзивных процессов.

1. Конформная симметрия лежит в основе важных теоретических исследований в различных безмассовых моделях квантовой теории поля [1], [2], включая квантовую электродинамику (КЭД) [3] и квантовую хромодинамику (КХД) (см. раздел 5 в работе [4]). Применение этой симметрии при изучении аксиальновекторной-векторной-векторной (АВВ) треугольной амплитуды позволило установить фундаментальное соотношение между важными характеристиками различных инклюзивных процессов [5]. В работе [5] исследуемыми характеристиками являются нормированные кварк-партонные приближения для несинглетной по аромату части $D_{\mathrm{A}}^{\mathrm{ns}}$ функции Адлера $D_{\mathrm{A}}$ процесса $e^{+} e^{-}$-аннигиляции в адроны и для несинглетной коэффициентной функции $C^{\text {Вjp }}$ правила сумм Бьеркена $S_{\text {Вjp }}$ процесса глубоконеупругого

* Институт ядерных исследований РАН, Москва, Россия. E-mail: kataev@ms2.inr.ac.ru

${ }^{\dagger}$ Объединенный институт ядерных исследований, Дубна, Московская обл., Россия. E-mail: mikhs@theor.jinr.ru 
рассеяния поляризованных лептонов на нуклонах, которая также входит в несинглетную часть правила сумм Эллиса-Джаффе глубоконеупругого рассеяния поляризованных лептонов на нуклонах.

Это основное соотношение Крютера было вскоре применено в работе [6] в модельном случае, когда в КЭД не учитываются диаграммы с лептонными вставками во внутренние фотонные линии. Соотношение также справедливо в умозрительном конформно-инвариантном пределе КХД. В этих случаях оно имеет вид

$$
\left.D \cdot C^{\mathrm{Bjp}}\right|_{\mathrm{ci}}=1
$$

где величины в его левой части определены как

$$
\begin{aligned}
D_{\mathrm{A}}^{\mathrm{ns}}\left(a_{\mathrm{s}}\right) & =\left(N_{\mathrm{c}} \sum_{f} Q_{f}^{2}\right) D\left(a_{\mathrm{s}}\right), \\
S_{\mathrm{Bjp}}\left(a_{\mathrm{s}}\right) & =\left(\frac{1}{6} \frac{g_{\mathrm{A}}}{g_{\mathrm{V}}}\right) C^{\mathrm{Bjp}}\left(a_{\mathrm{s}}\right) .
\end{aligned}
$$

Известно, что в моделях квантовой теории поля конформная симметрия нарушается перенормировками зарядов. Такие перенормировки приводят к существованию ненулевых ренормгрупповых (РГ) $\beta$-функций (детальное изложение см. в [7]). Более того, фактор $\beta\left(a_{\mathrm{s}}\right) / a_{\mathrm{s}}$, где $a_{\mathrm{s}}=\alpha_{\mathrm{s}} / \pi$, возникает в результате перенормировки следа тензора энергии-импульса. Это свойство было в общих чертах сформулировано теоретически в работе [8] и показано явно в [9]-[12]. Оно связано с существованием конформной аномалии.

До появления работы [13] не было ясно, существует ли обобщение основного соотношения Крютера [5] на случай калибровочных теорий с фермионами, таких как КЭД и КХД, в высших порядках теории возмущений (ТВ) при явном проявлении перенормировки константы связи. В работе [13] были классифицированы групповые множители группы $S U\left(N_{\mathrm{c}}\right)$, возникающие при умножении ряда ТВ КХД для функции $D^{\mathrm{ns}}$ в приближении $O\left(a_{\mathrm{s}}^{3}\right)$, полученном аналитически в работе [14], и позднее в [15] в схеме $\overline{\mathrm{MS}}$, на аналогичное приближение для функции $C^{\mathrm{Bjp}}$, известное к тому времени из расчетов работы [16]. Проведенные в работе [13] исследования позволили найти дополнительный вклад в правую часть выражения (1):

$$
D\left(a_{\mathrm{s}}\right) C^{\mathrm{Bjp}}\left(a_{\mathrm{s}}\right)=1+\Delta_{\mathrm{csb}}\left(a_{\mathrm{s}}\right) .
$$

В третьем порядке ТВ “единица Крютера” модифицируется нарушающим конформную симметрию членом $\Delta_{\mathrm{csb}}$, который представим в виде

$$
\Delta_{\mathrm{csb}}\left(a_{\mathrm{s}}\right)=\left(\frac{\beta\left(a_{\mathrm{s}}\right)}{a_{\mathrm{s}}}\right) P\left(a_{\mathrm{s}}\right)=\left(\frac{\beta\left(a_{\mathrm{s}}\right)}{a_{\mathrm{s}}}\right) \sum_{m \geqslant 1} K_{m} a_{\mathrm{s}}^{m} .
$$

В этом порядке ТВ в фактор $\beta\left(a_{\mathrm{s}}\right) / a_{\mathrm{s}}$ входит не зависящая от схемы двухпетлевая РГ $\beta$-функция, а в множитель $P\left(a_{\mathrm{s}}\right)$ - определенные в работе [13] коэффициенты $K_{1}$ и $K_{2}$, причем $K_{2}$ зависит от квадратичных операторов Казимира $C_{\mathrm{F}}, C_{\mathrm{A}}$ калибровочной группы $S U\left(N_{\mathrm{c}}\right)$ и от числа ароматов фермионов $n_{\mathrm{f}}$. 
Открытие такого КХД-обобщения соотношения Крютера в третьем порядке ТВ [13] в схеме $\overline{\mathrm{MS}}$ с фактором $\beta\left(a_{\mathrm{s}}\right) / a_{\mathrm{s}}$ явилось первым независимым теоретическим указанием на справедливость компьютерных аналитических вычислений поправки $O\left(a_{\mathrm{s}}^{3}\right)$ в ряде ТВ для $D$-функции [14], [15] и аналогичных вычислений третьего члена ряда ТВ для $C^{\text {Вjp }}[16]$. Отметим, что в дальнейшем это “проверочное” теоретическое указание было подтверждено независимыми компьютерными расчетами вклада порядка $a_{\mathrm{s}}^{3}$ в $D$-функцию, проведенными в работе [17] с помощью иного теоретического подхода.

Следующие за лидирующими поправки ТВ к $D$-функции были ранее вычислены аналитически [18] и численно [19]. Эти результаты были вскоре подтверждены аналитически [20]. В случае $C^{\text {Вjр }}$ поправки того же порядка ТВ были получены в работе [21] и подтверждены позднее [22] с применением другой символьной вычислительной техники.

Для объяснения происхождения эффекта факторизации $\beta\left(a_{\mathrm{s}}\right) / a_{\mathrm{s}}$ в $(5)$ в работе [23] к треугольной диаграмме ABВ-токов был применен метод операторного разложения в импульсном пространстве (более детальное обсуждение см. в [24]). В работе [23] также были приведены аргументы в пользу отсутствия противоречия между однопетлевой природой аксиальной аномалии [25] и многопетлевой структурой обобщения соотношения Крютера в КХД, заключающиеся в их связи с различными формфакторами в операторном разложении треугольной диаграммы ABВ-токов [23]. Было также указано на возможность факторизации многопетлевого множителя $\beta\left(a_{\mathrm{s}}\right) / a_{\mathrm{s}}$ при нарушающем конформную симметрию вкладе в выражении (5) во всех порядках ТВ [23] с коэффициентами $K_{m}$ полинома $P\left(a_{\mathrm{s}}\right)$, не фиксируемыми из проведенных рассмотрений. При этом подразумевалось использование схемы перенормировок $\overline{\mathrm{MS}}$, в которой можно явно определить коэффициентные функции лидирующего оператора в методе операторного разложения. Второй из сделанных в работе [23] выводов был доказан в координатном пространстве в работе [26] и обсуждался ранее [27], но был опубликован лишь недавно [4].

Более феноменологически ориентированное рассмотрение этого варианта КХДобобщения соотношения Крютера [13] в схеме $\overline{\mathrm{MS}}$ проведено в работах [28] и [29], где для фиксации характерного масштаба энергий использовалась разработанная в [30] многопетлевая версия подхода Бродского-Лепажа-Маккензи [31], дополненная процедурой построения "соразмерных масштабов" [32]. Результатом явилось “восстановление" в порядке $O\left(a_{\mathrm{s}}^{3}\right)$ "единицы Крютера" в правой части соотношения (4) путем включения нарушающего конформную симметрию члена в энергетический масштаб эффективного заряда для $D$-функции, что эквивалентно выбору некоторого масштаба в инвариантном заряде для $D$-функции, и включения нарушающего конформную симметрию члена в энергетический масштаб эффективного заряда для $C^{\text {Bjp }}[29]$.

В случае группы $S U\left(N_{\mathrm{c}}\right)$ поправки $O\left(a_{\mathrm{s}}^{4}\right)$ к функциям $D^{\mathrm{ns}}$ и $C^{\mathrm{Bjp}}\left(a_{\mathrm{s}}\right)$ известны благодаря недавним аналитическим расчетам из работы [33], проведенным в схеме $\overline{\mathrm{MS}}$. Данные вычисления с учетом трехпетлевых вкладов в $\beta$-функцию в этой схеме [34], [35] позволили фиксировать коэффициент $K_{3}$ в полиноме $P\left(a_{\mathrm{s}}\right)$ в выражении (5) и показать справедливость результатов работы [28] при учете $O\left(a_{\mathrm{s}}^{4}\right)$-вкладов ТВ. За-

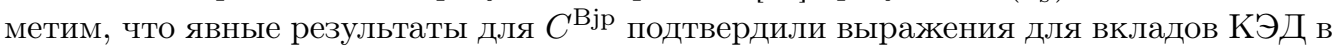


правило сумм Бьеркена, содержащих $\zeta_{3}$, которые впервые появились в работе [36] в поправках четвертого порядка квантово-электродинамической ТВ в $D^{\text {ns }}$-функции. Этот член в правиле сумм Бьеркена ранее был получен в [37] на основе результатов работы [36] с использованием конформной симметрии и основного соотношения Крюйтера ${ }^{1)}$. Найденное согласие явилось первым подтверждением справедливости расчетов работы [33].

Основная цель настоящей работы состоит в обосновании детального представления обобщенного соотношения Крютера в порядке $a_{\mathrm{s}}^{4}$, предложенного нами ранее [39]. Его новизна заключается в записи правой части соотношения (5) в виде двойного степенного разложения, в котором первым параметром разложения является функция $\beta\left(a_{\mathrm{s}}\right) / a_{\mathrm{s}}$, а вторым - константа связи $a_{\mathrm{s}}$, а именно

$$
\begin{aligned}
\Delta_{\mathrm{csb}}\left(a_{\mathrm{s}}\right) & =\sum_{n \geqslant 1}\left(\frac{\beta\left(a_{\mathrm{s}}\right)}{a_{\mathrm{s}}}\right)^{n} \mathcal{P}_{n}\left(a_{\mathrm{s}}\right)=\sum_{n \geqslant 1} \sum_{r \geqslant 1}\left(\frac{\beta\left(a_{\mathrm{s}}\right)}{a_{\mathrm{s}}}\right)^{n} P_{n}^{(r)} a_{\mathrm{s}}^{r}= \\
& =\sum_{n \geqslant 1} \sum_{r \geqslant 1}\left(\frac{\beta\left(a_{\mathrm{s}}\right)}{a_{\mathrm{s}}}\right)^{n} P_{n}^{(r)}[k, m] C_{\mathrm{F}}^{k} C_{\mathrm{A}}^{m} a_{\mathrm{s}}^{r},
\end{aligned}
$$

где $k+m=r$ и коэффициенты $P_{n}^{(r)}[k, m]$ содержат рациональные дроби и дзета-функции Римана от нечетных целых чисел. В отличие от коэффициентов полинома $P\left(a_{\mathrm{s}}\right)$ в выражении (5), коэффициенты $\mathcal{P}_{n}\left(a_{\mathrm{s}}\right)$ в соотношении (6) уже не зависят от числа ароматов кварков $n_{\mathrm{f}}$.

2. Рассмотрим ряды ТВ для определенных в (2) и (3) нормированных на единицу несинглетной части функции Адлера $D$ и коэффициентной функции правила сумм Бьеркена $C^{\text {Bjp }}$ :

$$
D=1+\sum_{n=1} d_{n} a_{\mathrm{s}}^{n}, \quad C^{\mathrm{Bjp}}=1+\sum_{l=1} c_{l} a_{\mathrm{s}}^{l} .
$$

Явные выражения для $d_{1}, d_{2}, d_{3}$ и $c_{1}, c_{2}, c_{3}$ через групповые факторы $S U\left(N_{\mathrm{c}}\right)$ хорошо известны (см., например, [14], [16]). В конкретном случае группы $S U(3)$ четвертый коэффициент $D$-функции $d_{4}$ был аналитически вычислен в работе [40]. Недавно этот результат был обобщен на случай произвольной цветовой группы $S U\left(N_{\mathrm{c}}\right)$ [33]. Аналогичный коэффициент $c_{4}$ для $C^{\text {Вjp }}$, также вычисленный в работе [33] (см. приложенный к версии статьи в архиве препринтов отдельный файл), имеет вид ${ }^{2)}$

$$
\begin{aligned}
c_{4}= & \left.-\frac{3}{16}+\frac{1}{4} \zeta_{3}+\frac{5}{4} \zeta_{5}\right] \frac{d_{\mathrm{F}}^{a b c d} d_{\mathrm{A}}^{a b c d}}{d_{\mathrm{R}}}+\left[\frac{13}{16}+\zeta_{3}-\frac{5}{2} \zeta_{5}\right] \frac{d_{\mathrm{F}}^{a b c d} d_{\mathrm{F}}^{a b c d}}{d_{\mathrm{R}}} n_{\mathrm{f}}- \\
& -\left[\frac{4823}{2048}+\frac{3}{8} \zeta_{3}\right] C_{\mathrm{F}}^{4}+\left[\frac{839}{2304}+\frac{451}{96} \zeta_{3}-\frac{145}{24} \zeta_{5}\right] C_{\mathrm{F}}^{3} T_{\mathrm{F}} n_{\mathrm{f}}+
\end{aligned}
$$

\footnotetext{
1) Аргументы в пользу возможного объяснения появления на этом уровне ТВ $\zeta_{3}$-члена, нетипичного для известных ранее вкладов диаграмм, характеризующих фотонную функцию поляризации вакуума в КЭД без фермионных вставок во внутренние фотонные линии, приведены в работе [38].

2)Это выражение, согласующееся с результатом в электронном приложении к препринту работы [33], было восстановлено нами из ее текста, в котором приведен результат для величины $1 / C^{\mathrm{Bjp}}$.
} 


$$
\begin{aligned}
& +\left[-\frac{265}{576}+\frac{29}{24} \zeta_{3}\right] C_{\mathrm{F}}^{2} T_{\mathrm{F}}^{2} n_{\mathrm{f}}^{2}+\frac{605}{972} C_{\mathrm{F}} T_{\mathrm{F}}^{3} n_{\mathrm{f}}^{3}+ \\
& +\left[-\frac{3707}{4608}-\frac{971}{96} \zeta_{3}+\frac{1045}{48} \zeta_{5}\right] C_{\mathrm{F}}^{3} C_{\mathrm{A}}+ \\
& +\left[-\frac{87403}{13824}-\frac{1289}{144} \zeta_{3}+\frac{275}{144} \zeta_{5}+\frac{35}{4} \zeta_{7}\right] C_{\mathrm{F}}^{2} C_{\mathrm{A}} T_{\mathrm{F}} n_{\mathrm{f}}+ \\
& +\left[-\frac{165283}{20736}-\frac{43}{144} \zeta_{3}+\frac{5}{12} \zeta_{5}-\frac{1}{6} \zeta_{3}^{2}\right] C_{\mathrm{F}} C_{\mathrm{A}} T_{\mathrm{F}}^{2} n_{\mathrm{f}}^{2}+ \\
& +\left[\frac{1071641}{55296}+\frac{1591}{144} \zeta_{3}-\frac{1375}{144} \zeta_{5}-\frac{385}{16} \zeta_{7}\right] C_{\mathrm{F}}^{2} C_{\mathrm{A}}^{2}+ \\
& +\left[\frac{1238827}{41472}+\frac{59}{64} \zeta_{3}-\frac{1855}{288} \zeta_{5}+\frac{11}{12} \zeta_{3}^{2}-\frac{35}{16} \zeta_{7}\right] C_{\mathrm{F}} C_{\mathrm{A}}^{2} T_{\mathrm{F}} n_{\mathrm{f}}+ \\
& +\left[-\frac{8004277}{248832}+\frac{1069}{576} \zeta_{3}+\frac{12545}{1152} \zeta_{5}-\frac{121}{96} \zeta_{3}^{2}+\frac{385}{64} \zeta_{7}\right] C_{\mathrm{F}} C_{\mathrm{A}}^{3},
\end{aligned}
$$

где $\zeta_{2 q+1}=\sum_{k=1}^{\infty}(1 / k)^{2 q+1}-$ функции Римана нечетных аргументов. В фундаментальном представлении группы $S U\left(N_{\mathrm{c}}\right)$ выражения для групповых факторов определяются как $C_{\mathrm{F}}=\left(N_{\mathrm{c}}^{2}-1\right) /\left(2 N_{\mathrm{c}}\right), C_{\mathrm{A}}=N_{\mathrm{c}}, T_{\mathrm{F}}=1 / 2, d_{\mathrm{F}}^{a b c d} d_{\mathrm{A}}^{a b c d} / d_{\mathrm{R}}=$ $N_{\mathrm{c}}\left(N_{\mathrm{c}}^{2}+6\right) / 18, d_{\mathrm{F}}^{a b c d} d_{\mathrm{F}}^{a b c d} / d_{\mathrm{R}}=\left(N_{\mathrm{c}}^{4}-6 N_{\mathrm{c}}^{2}+18\right) /\left(36 N_{\mathrm{c}}^{2}\right)$. Для группы $S U(3)$, что соответствует случаю КХД, имеем $C_{\mathrm{F}}=4 / 3, C_{\mathrm{A}}=3, d_{\mathrm{R}}=3$ и $d_{\mathrm{F}}^{a b c d} d_{\mathrm{A}}^{a b c d}=15 / 2$, $d_{\mathrm{F}}^{a b c d} d_{\mathrm{F}}^{a b c d}=5 / 12$.

Сильная проверка самосогласованности результатов, полученных в работах [40] и [33], следует из справедливости КХД-обобщения соотношения Крютера (5) при учете вкладов в левой части определения (4) поправок $O\left(a_{\mathrm{s}}^{4}\right)$, вычисленных в схеме $\overline{\mathrm{MS}}$. Напомним, что существование подобного обобщения с факторизацией множителя $\beta\left(a_{\mathrm{s}}\right) / a_{\mathrm{s}}$ не случайно. Оно было обнаружено в предыдущем порядке ТВ [13] и доказано во всех порядках в работе [26]. В работе [13] было показано, что в третьем порядке ТВ выражения для коэффициентов полинома $P\left(a_{\mathrm{s}}\right)$ в $(5)$ могут быть записаны как

$$
\begin{aligned}
& K_{1}=K_{1}[1,0,0] C_{\mathrm{F}} \\
& K_{2}=K_{2}[2,0,0] C_{\mathrm{F}}^{2}+K_{2}[1,1,0] C_{\mathrm{F}} C_{\mathrm{A}}+K_{2}[1,0,1] C_{\mathrm{F}} T_{\mathrm{F}} n_{\mathrm{f}}
\end{aligned}
$$

Проведенные в [33] вычисления в четвертом порядке ТВ приводят к фиксации третьего члена полинома $P\left(a_{\mathrm{s}}\right)$ в виде суммы шести слагаемых, пропорциональных произведению операторов Казимира группы $S U\left(N_{\mathrm{c}}\right)$ и числа ароматов кварков $n_{\mathrm{f}}$ :

$$
\begin{gathered}
K_{3}=K_{3}[3,0,0] C_{\mathrm{F}}^{3}+K_{3}[2,1,0] C_{\mathrm{F}}^{2} C_{\mathrm{A}}+K_{3}[1,2,0] C_{\mathrm{F}} C_{\mathrm{A}}^{2}+K_{3}[2,0,1] C_{\mathrm{F}}^{2} T_{\mathrm{F}} n_{\mathrm{f}}+ \\
+K_{3}[1,1,1] C_{\mathrm{F}} C_{\mathrm{A}} T_{\mathrm{F}} n_{\mathrm{f}}+K_{3}[1,0,2] C_{\mathrm{F}}\left(T_{\mathrm{F}} n_{\mathrm{f}}\right)^{2}
\end{gathered}
$$

Аналитическое выражение для последнего коэффициента $K_{3}[1,0,2]$ в $(10)$ совпадает с результатом работы [13], полученным в процессе вычислений аналогичных коэф- 
фициентов, генерируемых в высших порядках ТВ произведением вкладов в функции $D\left(a_{\mathrm{s}}\right)$ и $C^{\mathrm{Bjp}}$ диаграмм с большим числом фермионных вставок во внутренние глюонные линии.

В соответствии со структурой члена $\Delta_{\mathrm{csb}}\left(a_{\mathrm{s}}\right)$ в соотношениях $(5)$ и $(6)$ нам потребуются конкретные значения коэффициентов РГ $\beta$-функции схемы $\overline{\mathrm{MS}}$

$$
\mu^{2} \frac{d}{d \mu^{2}} a_{\mathrm{s}}=\beta\left(a_{\mathrm{s}}\right)=-a_{\mathrm{s}}^{2}\left(\beta_{0}+\beta_{1} a_{\mathrm{s}}+\beta_{2} a_{\mathrm{s}}^{2}\right)
$$

найденных в трехпетлевом приближении в [34] и подтвержденных в [35]. Коэффициенты $\beta_{i}$ могут быть представлены в виде

$$
\begin{aligned}
& \beta_{0}=\beta_{0}[0,1,0] C_{\mathrm{A}}+\beta_{0}[0,0,1] T_{\mathrm{F}} n_{\mathrm{f}}, \\
& \beta_{1}=\beta_{1}[0,2,0] C_{\mathrm{A}}^{2}+\beta_{1}[0,1,1] C_{\mathrm{A}} T_{\mathrm{F}} n_{\mathrm{f}}+\beta_{1}[1,0,1] C_{\mathrm{F}} T_{\mathrm{F}} n_{\mathrm{f}}, \\
& \beta_{2}=\beta_{2}[0,3,0] C_{\mathrm{A}}^{3}+\beta_{2}[0,2,1] C_{\mathrm{A}}^{2} T_{\mathrm{F}} n_{\mathrm{f}}+\beta_{2}[1,1,1] C_{\mathrm{F}} C_{\mathrm{A}} T_{\mathrm{F}} n_{\mathrm{f}}+ \\
& \quad+\beta_{2}[0,1,2] C_{\mathrm{A}} T_{\mathrm{F}}^{2} n_{\mathrm{f}}^{2}+\beta_{2}[2,0,1] C_{\mathrm{F}}^{2} T_{\mathrm{F}} n_{\mathrm{f}}+\beta_{2}[1,0,2] C_{\mathrm{F}} T_{\mathrm{F}}^{2} n_{\mathrm{f}}^{2}
\end{aligned}
$$

со следующими элементами $\beta_{j}[\ldots]$ :

$$
\begin{aligned}
& \beta_{0}[0,1,0]=\frac{11}{12}, \quad \beta_{0}[0,0,1]=-\frac{1}{3}, \\
& \beta_{1}[0,2,0]=\frac{17}{24}, \quad \beta_{1}[0,1,1]=-\frac{5}{12}, \quad \beta_{1}[1,0,1]=-\frac{1}{4}, \\
& \beta_{2}[0,3,0]=\frac{2857}{3456}, \quad \beta_{2}[0,2,1]=-\frac{1415}{1728}, \quad \beta_{2}[1,1,1]=-\frac{205}{576}, \\
& \beta_{2}[0,1,2]=\frac{79}{864}, \quad \beta_{2}[2,0,1]=\frac{1}{32}, \quad \beta_{2}[1,0,2]=\frac{11}{144} .
\end{aligned}
$$

3. Рассмотрим теперь вопрос об однозначности детального обобщения соотношения Крютера (6) по степеням $\beta$-функции. Мы приведем здесь дополнительные соображения в пользу нашего предположения о существовании такого обобщения (см. [39]) и представим его обоснование с использованием полученного в работе [33] приближения четвертого порядка ТВ для выражений (4), (5).

Вывод детального обобщения соотношения Крютера в схеме $\overline{\mathrm{MS}}$ основывается на требовании независимости коэффициентов полинома $\mathcal{P}_{n}$ в (6) от коэффициентов $\beta$-функции, а следовательно, и от числа ароматов кварков $n_{\mathrm{f}}$. Это свойство может быть реализовано путем перехода в нарушающем конформную симметрию члене $\Delta_{\mathrm{csb}}\left(a_{\mathrm{s}}\right)$ выражения (4) от представления (5) с единственной факторизованной $\beta$-функцией к представлению (6) в виде разложения по степеням $\beta\left(a_{\mathrm{s}}\right) / a_{\mathrm{s}}$. Предположение о справедливости такой формы записи члена $\Delta_{\mathrm{csb}}\left(a_{\mathrm{s}}\right)$ в четвертом порядке ТВ было сделано в работе [39] до публикации результатов в порядке $a_{\mathrm{s}}^{4}$ аналитических расчетов $D$-функции и $C^{\mathrm{Bjp}}\left(a_{\mathrm{s}}\right)[33]$. Для ее явного вывода следует приравнять правые части двух представлений члена $\Delta_{\mathrm{csb}}\left(a_{\mathrm{s}}\right)$ из соотношений $(5)$ и $(6)$ в каждом порядке разложения по константе связи $a_{\mathrm{s}}$. В интересующем нас приближении ТВ коэффициенты в правой части соотношения (5) связаны с аналогичными вкладами 
в соотношения (6) следующей системой линейных уравнений:

$$
\begin{aligned}
K_{1}[1,0,0]= & P_{1}^{(1)}[1,0], \\
K_{2}[2,0,0]= & P_{1}^{(2)}[2,0], \\
K_{2}[1,1,0]= & P_{1}^{(2)}[1,1]-\beta_{0}[0,1,0] P_{2}^{(1)}[1,0], \\
K_{2}[1,0,1]= & -\beta_{0}[0,0,1] P_{2}^{(1)}[1,0], \\
K_{3}[3,0,0]= & P_{1}^{(3)}[3,0], \\
K_{3}[2,1,0]= & P_{1}^{(3)}[2,1]-\beta_{0}[0,1,0] P_{2}^{(2)}[2,0], \\
K_{3}[1,2,0]= & P_{1}^{(3)}[1,2]-\beta_{0}[0,1,0] P_{2}^{(2)}[1,1]-\beta_{1}[0,2,0] P_{2}^{(1)}[1,0]+ \\
& +\left(\beta_{0}[0,1,0]\right)^{2} P_{3}^{(1)}[1,0], \\
K_{3}[2,0,1]= & -\beta_{1}[1,0,1] P_{2}^{(1)}[1,0]-\beta_{0}[0,0,1] P_{2}^{(2)}[2,0], \\
K_{3}[1,1,1]= & -\beta_{1}[0,1,1] P_{2}^{(1)}[1,0]-\beta_{0}[0,0,1] P_{2}^{(2)}[1,1]+ \\
& +2 \beta_{0}[0,1,0] \beta_{0}[0,0,1] P_{3}^{(1)}[1,0], \\
K_{3}[1,0,2]= & \left(\beta_{0}[0,0,1]\right)^{2} P_{3}^{(1)}[1,0] .
\end{aligned}
$$

Однозначные решения этой системы определяют явные выражения для коэффициентов трех полиномов $\mathcal{P}_{n}\left(a_{\mathrm{s}}\right)$ с не зависящими от числа ароматов $n_{\mathrm{f}}$ коэффициентами $P_{n}^{(r)}[k, m]$ :

$$
\begin{aligned}
\mathcal{P}_{1}\left(a_{\mathrm{s}}\right)=( & \left.-\frac{21}{8}+3 \zeta_{3}\right) C_{\mathrm{F}} a_{\mathrm{s}}+\left[\left(\frac{397}{96}+\frac{17}{2} \zeta_{3}-15 \zeta_{5}\right) C_{\mathrm{F}}^{2}+\right. \\
& \left.+\left(-\frac{47}{48}+\zeta_{3}\right) C_{\mathrm{F}} C_{\mathrm{A}}\right] a_{\mathrm{s}}^{2}+\left[\left(\frac{2471}{768}+\frac{61}{8} \zeta_{3}-\frac{715}{8} \zeta_{5}+\frac{315}{4} \zeta_{7}\right) C_{\mathrm{F}}^{3}+\right. \\
& +\left(\frac{16649}{1536}-\frac{11183}{192} \zeta_{3}+\frac{1015}{24} \zeta_{5}-\frac{105}{8} \zeta_{7}+\frac{99}{4} \zeta_{3}^{2}\right) C_{\mathrm{F}}^{2} C_{\mathrm{A}}+ \\
& \left.+\left(\frac{2107}{192}+\frac{2503}{72} \zeta_{3}-\frac{355}{18} \zeta_{5}-33 \zeta_{3}^{2}\right) C_{\mathrm{F}} C_{\mathrm{A}}^{2}\right] a_{\mathrm{s}}^{3}+O\left(a_{\mathrm{s}}^{4}\right), \\
\mathcal{P}_{2}\left(a_{\mathrm{s}}\right)=( & \left.\frac{163}{8}-19 \zeta_{3}\right) C_{\mathrm{F}} a_{\mathrm{s}}+\left[\left(-\frac{13597}{384}-\frac{2523}{16} \zeta_{3}+\frac{375}{2} \zeta_{5}+27 \zeta_{3}^{2}\right) C_{\mathrm{F}}^{2}+\right. \\
& \left.+\left(\frac{1433}{32}-\frac{1}{4} \zeta_{3}-\frac{85}{2} \zeta_{5}-6 \zeta_{3}^{2}\right) C_{\mathrm{F}} C_{\mathrm{A}}\right] a_{\mathrm{s}}^{2}+O\left(a_{\mathrm{s}}^{3}\right), \\
\mathcal{P}_{3}\left(a_{\mathrm{s}}\right)=( & \left.-\frac{307}{2}+\frac{203}{2} \zeta_{3}+45 \zeta_{5}\right) C_{\mathrm{F}} a_{\mathrm{s}}+O\left(a_{\mathrm{s}}^{2}\right) .
\end{aligned}
$$

Отметим, что в четырехпетлевом члене $\beta_{3} \mathrm{P} \Gamma \beta$-функции, вычисленном аналитически в работе [41] в случае группы $S U\left(N_{\mathrm{c}}\right)$ и позднее подтвержденном в [42], появляются три новые групповые структуры $d_{\mathrm{A}}^{a b c d} d_{\mathrm{A}}^{a b c d}, d_{\mathrm{F}}^{a b c d} d_{\mathrm{A}}^{a b c d} n_{\mathrm{f}}$ и $d_{\mathrm{F}}^{a b c d} d_{\mathrm{F}}^{a b c d} n_{\mathrm{f}}^{2}$. В силу факторизации $\beta$-функции в (5) во всех порядках ТВ (см. доказательства в работах [26], [4]) делаем вывод, что появление этих дополнительных групповых структур не разрушит свойство факторизации четырехпетлевого приближения $\beta$-функции как в (5), так и в первом члене суммы в (6). 
Еще один вывод следует из выполненных в работе [13] вычислений высших вкладов по степеням $n_{\mathrm{f}}$, эквивалентных вычислению поправок, пропорциональных высшим степеням первого коэффициента РГ $\beta$-функции $\beta_{0}$. Эти поправки определяют лидирующие вклады в полиномы $\mathcal{P}_{n}\left(a_{\mathrm{s}}\right)$ нового представления для члена $\Delta_{\mathrm{csb}}$ в $(6)$, которые имеют вид

$$
\mathcal{P}_{n}\left(a_{\mathrm{s}}\right)=\frac{S_{n}}{4^{n}} 3^{(n-1)} C_{\mathrm{F}} a_{\mathrm{s}}+O\left(a_{\mathrm{s}}^{2}\right) .
$$

При этом первые девять коэффициентов $S_{n}, 1 \leqslant n \leqslant 9$, вычислены аналитически в работе [13].

4. Представление (6) может быть получено другим способом с применением развитого в работе [43] формализма $\beta$-разложений коэффициентов рядов ТВ (в схеме $\overline{\mathrm{MS}})$. В этом подходе предлагается рассматривать разложение коэффициентов $d_{n}$ и $c_{n}$ по степеням коэффициентов $\beta$-функции $\beta_{0}, \beta_{1}, \ldots$ с весовыми элементами $d_{n}\left[n_{0}, n_{1}, \ldots\right], c_{n}\left[n_{0}, n_{1}, \ldots\right]$ вместо общепринятого разложения коэффициентов по степеням зависящего от аромата фактора $T_{\mathrm{F}} n_{\mathrm{f}}$, квадратичным операторам Казимира $C_{\mathrm{F}}$ и $C_{\mathrm{A}}$ и структурным константам цветовой группы $S U\left(N_{\mathrm{c}}\right)$. Их первые аргументы $\left(n_{0}\right)$ определяют степени коэффициентов $\beta_{0}$ при элементах $d_{n}[\cdot]$ и $c_{n}[\cdot]$, вторые аргументы $\left(n_{1}\right)$ дают степени коэффициентов $\beta_{1}$ и т. д. Элементы $d_{n}[0,0, \ldots, 0], c_{n}[0,0, \ldots, 0]$ являются вкладами, "очищенными" от перенормировок заряда и множителей $\beta_{i}$, степени которых здесь равны нулю $\left(n_{i}=0\right)$. Эти элементы совпадают со значениями коэффициентов $d_{n}, c_{n}$ в гипотетическом пределе с $\beta$-функцией, тождественно равной нулю во всех порядках ТВ в КХД. Такой предел соответствует восстановлению конформной симметрии в эффективной квантово-полевой модели, переход к нему будет рассматриваться здесь как технический прием. В случае, когда все аргументы $n_{i}$ элементов $d_{n}[\ldots, m, 0, \ldots, 0]$, $c_{n}[\ldots, m, 0, \ldots, 0]$ после некоторого индекса $m$ равны нулю, упростим обозначения следующим образом: $d_{n}[\ldots, m, 0, \ldots, 0]=d_{n}[\ldots, m], c_{n}[\ldots, m, 0, \ldots, 0]=c_{n}[\ldots, m]$. Соответствующие $\beta$-представления для первых нескольких коэффициентов выражения (7) суть

$$
\begin{aligned}
d_{2}= & \beta_{0} d_{2}[1]+d_{2}[0] \\
d_{3}= & \beta_{0}^{2} d_{3}[2]+\beta_{1} d_{3}[0,1]+\beta_{0} d_{3}[1]+d_{3}[0] \\
d_{4}= & \beta_{0}^{3} d_{4}[3]+\beta_{1} \beta_{0} d_{4}[1,1]+\beta_{2} d_{4}[0,0,1]+\beta_{0}^{2} d_{4}[2]+ \\
& +\beta_{1} d_{4}[0,1]+\beta_{0} d_{4}[1]+d_{4}[0] .
\end{aligned}
$$

Аналогичные представления справедливы и для высших коэффициентов $d_{n}$ рядов ТВ для $D$-функции, и для коэффициентов $c_{l}$ ряда ТВ для $C^{\mathrm{Bjp}}$, определенных в $(7)$, и т. п. Подчеркнем, что представления вида (17)-(19) являются однозначными. Коэффициенты $d_{n}[n-1], c_{n}[n-1]$ совпадают с членами, генерируемыми цепочками одночастично-приводимых однопетлевых фермионных вставок в глюонные пропагаторы, и могут быть найдены, например, в работе [13]. Определение явного вида других элементов является отдельной непростой задачей. Их диаграммное представление обсуждалось в работе [43]. Ниже мы обсудим способ получения конкретных аналитических выражений для элементов коэффициентов $d_{n}$ и $c_{l}$ вплоть до поправок порядка $a_{\mathrm{s}}^{3}$.

Разложение (6) в совокупности с выражениями (17)-(19) позволяет найти соотношения между неизвестными элементами коэффициентов четвертого порядка ряда 
TВ $d_{4}, c_{4}$ и элементами, входящими в коэффициенты третьего порядка ряда ТВ (которые будут явно приведены ниже).

В силу соотношения (1), следующего из ненарушенной конформной симметрии, восстанавливаемой в гипотетическом случае $\beta_{i}=0$, находим явную связь между "очищенными" от перенормировок заряда вкладами:

$$
c_{n}[0]+d_{n}[0]+\sum_{l=1}^{n-1} d_{l}[0] c_{n-l}[0]=0 .
$$

Особенностью этого рекуррентного соотношения является возможность выразить сумму элементов $n$-го порядка ТВ через аналогичные элементы, входящие в коэффициенты низших приближений ТВ. Отсюда следует связь между "очищенными" элементами $c_{4}[0], d_{4}[0]$ коэффициентов четвертого порядка ТВ:

$$
c_{4}[0]+d_{4}[0]=2 d_{1} d_{3}[0]-3 d_{1}^{2} d_{2}[0]+\left(d_{2}[0]\right)^{2}+d_{1}^{4} .
$$

Отметим, что это соотношение содержит вклады не только $C_{\mathrm{F}}$, но также и $C_{\mathrm{A}}$. Напомним, что проекция выражения $(21)$ на максимальную степень Казимира $C_{\mathrm{F}}, C_{\mathrm{F}}^{4}$ эквивалентна соотношению, использовавшемуся ранее в работе [37] при формулировке предложения о проверке результата КЭД для аналога коэффициента $d_{4}$, впервые опубликованного в докладе [36].

Явное выражение для коэффицента $d_{3}$ в $\beta$-разложении было получено в работе [43] благодаря использованию вычисленного в [17] аналитического результата для вклада в третий коэффициент ряда ТВ функции Адлера $D\left(a_{\mathrm{s}}, n_{\mathrm{f}}, n_{\tilde{\mathrm{g}}}\right)$ поправок с числом $n_{\tilde{\mathrm{g}}}$ мультиплетов глюино в приближении, когда в суперсимметричном варианте КХД не учитываются вклады скалярных кварков (скварков). На уровне поправок порядка $a_{\mathrm{s}}^{2}$ приведенный в [17] аналитический результат для глюинного вклада совпадает с численным результатом работы [44], а глюинная поправка порядка $a_{\mathrm{s}}^{3}$, вычисленная аналитически в работе [17] в схеме $\overline{\mathrm{MS}}$, была подтверждена в работе [45].

Элемент $d_{3}[2]$ в выражении (18) получить несложно. Его значение можно также извлечь из результатов работы [13]. Затем следует разделить в выражении для $d_{3}$ вклады от членов $\beta_{1} d_{3}[0,1]$ и $\beta_{0} d_{3}[1]$. Оба они линейны по числу ароматов кварков $n_{\mathrm{f}}$. Они разделяются при использовании дополнительной степени свободы упомянутого выше глюинного вклада, помеченного числом глюинных мультиплетов $n_{\tilde{\mathrm{g}}}{ }^{3)}$.

При этом можно найти явный вид функций $n_{\mathrm{f}}=n_{\mathrm{f}}\left(\beta_{0}, \beta_{1}\right)$ и $n_{\tilde{\mathrm{g}}}=n_{\tilde{\mathrm{g}}}\left(\beta_{0}, \beta_{1}\right)$. Эти выражения могут быть получены после учета глюинных вкладов в первые два коэффициента $\beta$-функции для подобного расширения КХД. Эти двухпетлевые результаты известны из выполненных в работе [48] вычислений. Именно таким образом в работе [43] были получены определенные в формулах (17), (18) коэффициенты

\footnotetext{
3) Отметим, что возможность существования глюино, более легкого, чем скварки Минимальной стандартной суперсимметричной модели (МССМ), с массой в области $m_{\tilde{g}} \geqslant 195$ ГэВ не противоречила экспериментальным данным Тэватрона [46], но недавно была исключена данными, полученными на Большом адронном коллайдере (БАК). Однако совместный детальный анализ имеющихся данных БАК, полученных коллаборациями CMS и ATLAS, все еще не исключает возможности существования варианта MCCM с более тяжелым, чем скварки, глюино с массой в области $m_{\tilde{\mathrm{g}}} \geqslant 400$ ГэВ [47].
} 
$\beta$-разложения членов $d_{2}$ и $d_{3}$. Мы приводим здесь результаты работы [43], лишь несколько изменив нормировочные коэффициенты:

$$
\begin{aligned}
& d_{1}=\frac{3}{4} C_{\mathrm{F}}, \quad d_{2}[1]=\left(\frac{33}{8}-3 \zeta_{3}\right) C_{\mathrm{F}}, \quad d_{2}[0]=-\frac{3}{32} C_{\mathrm{F}}^{2}+\frac{1}{16} C_{\mathrm{F}} C_{\mathrm{A}}, \\
& d_{3}[2]=\left(\frac{151}{6}-19 \zeta_{3}\right) C_{\mathrm{F}}, \\
& d_{3}[1]=\left(-\frac{27}{8}-\frac{39}{4} \zeta_{3}+15 \zeta_{5}\right) C_{\mathrm{F}}^{2}-\left(\frac{9}{64}-5 \zeta_{3}+\frac{5}{2} \zeta_{5}\right) C_{\mathrm{F}} C_{\mathrm{A}}, \\
& d_{3}[0,1]=\left(\frac{101}{16}-6 \zeta_{3}\right) C_{\mathrm{F}}, \\
& d_{3}[0]=-\frac{69}{128} C_{\mathrm{F}}^{3}+\frac{71}{64} C_{\mathrm{F}}^{2} C_{\mathrm{A}}+\left(\frac{523}{768}-\frac{27}{8} \zeta_{3}\right) C_{\mathrm{F}} C_{\mathrm{A}}^{2} .
\end{aligned}
$$

Выразим теперь элементы $c_{3}[\cdot]$ в аналогичном виде. Применяя формулу $(20)$ для определения $c_{3}[0]$ и учитывая аналитическое значение для $d_{3}[0](24)$, получаем

$$
\begin{gathered}
c_{1}=-\frac{3}{4} C_{\mathrm{F}}, \quad c_{2}[1]=-\frac{3}{2} C_{\mathrm{F}}, \quad c_{2}[0]=\frac{21}{32} C_{\mathrm{F}}^{2}-\frac{1}{16} C_{\mathrm{F}} C_{\mathrm{A}}, \\
c_{3}[2]=-\frac{115}{24} C_{\mathrm{F}}, \quad c_{3}[1]=\left(\frac{83}{24}-\zeta_{3}\right) C_{\mathrm{F}}^{2}+\left(\frac{215}{192}-6 \zeta_{3}+\frac{5}{2} \zeta_{5}\right) C_{\mathrm{F}} C_{\mathrm{A}}, \\
c_{3}[0,1]=\left(-\frac{59}{16}+3 \zeta_{3}\right) C_{\mathrm{F}}, \quad c_{3}[0]=-\frac{3}{128} C_{\mathrm{F}}^{3}-\frac{65}{64} C_{\mathrm{F}}^{2} C_{\mathrm{A}}-\left(\frac{523}{768}-\frac{27}{8} \zeta_{3}\right) C_{\mathrm{F}} C_{\mathrm{A}}^{2} .
\end{gathered}
$$

Разложения, аналогичные формулам (17), (18), были использованы ранее в работе [49] как для функции Адлера, так и для правила сумм Бьеркена. Однако при этом учитывались лишь члены, пропорциональные степеням $\beta_{0}$ (включая нулевую степень). В целом более последовательно использовать подход работы [43], который предписывает учитывать также вклад двухпетлевого коэффициента $\beta_{1}$ в РГ $\beta$-функции.

Подставляя теперь соотношения вида (17)-(19) для $d_{i}$ и $c_{i}$ в предложенное нами представление (6), приходим к следующим выражениям:

$$
\begin{aligned}
\mathcal{P}_{1}\left(a_{\mathrm{s}}\right)= & a_{\mathrm{s}}\left\{P_{1}^{(1)}+a_{\mathrm{s}} P_{1}^{(2)}+a_{\mathrm{s}}^{2} P_{1}^{(3)}\right\}= \\
= & -a_{\mathrm{s}}\left\{c_{2}[1]+d_{2}[1]+a_{\mathrm{s}}\left(c_{3}[1]+d_{3}[1]+d_{1}\left(c_{2}[1]-d_{2}[1]\right)\right)+\right. \\
& \left.\quad+a_{\mathrm{s}}^{2}\left(c_{4}[1]+d_{4}[1]+d_{1}\left(c_{3}[1]-d_{3}[1]\right)+d_{2}[0] c_{2}[1]+d_{2}[1] c_{2}[0]\right)\right\}, \\
\mathcal{P}_{2}\left(a_{\mathrm{s}}\right)= & a_{\mathrm{s}}\left\{P_{2}^{(1)}+a_{\mathrm{s}} P_{2}^{(2)}\right\}= \\
= & a_{\mathrm{s}}\left\{c_{3}[2]+d_{3}[2]+a_{\mathrm{s}}\left(c_{4}[2]+d_{4}[2]-d_{1}\left(c_{3}[2]-d_{3}[2]\right)\right)\right\}, \\
\mathcal{P}_{3}\left(a_{\mathrm{s}}\right)= & a_{\mathrm{s}} P_{3}^{(1)}=-a_{\mathrm{s}}\left\{c_{4}[3]+d_{4}[3]\right\}=a_{\mathrm{s}} C_{\mathrm{F}}\left(\frac{307}{2}-\frac{203}{2} \zeta_{3}-45 \zeta_{5}\right), \\
\mathcal{P}_{n}\left(a_{\mathrm{s}}\right)= & a_{\mathrm{s}} P_{n}^{(1)}=(-1)^{n-1} a_{\mathrm{s}}\left\{c_{n}[n-1]+d_{n}[n-1]\right\} .
\end{aligned}
$$

Конкретное выражение для формулы (29) определено в (16). Подчеркнем, что полученный ранее аналитический вид формул (15) приобретает конкретную связь с методом $\beta$-разложения (см. формулы $(26)-(28))$. Элементы $d_{n}[n-1]\left(c_{n}[n-1]\right)$ 
определяются диаграммами, содержащими единственный глюонный пропагатор с цепочкой одночастично-приводимых однопетлевых фермионных вставок (так называемые лидирующие ренормалонные вклады), и могут быть определены из результатов, полученных в [13]. Элементы $d_{n}[l], l<n-1$, определяются диаграммами, которые содержат по крайней мере два глюонных пропагатора, причем оба содержат одночастично-приводимые однопетлевые фермионные вставки (так называемые нелидирующие ренормалонные вклады). Подобные классы диаграмм до сих пор явно не вычислялись. Используя основной теоретический результат (6), явно проверенный нами в четвертом порядке ТВ, можно получить соотношения между элементами $\beta$-разложений коэффициентов $d_{4}\left(d_{n}\right)$ и $c_{4}\left(c_{n}\right)$. Так, первый член полинома $\mathcal{P}_{1}\left(a_{\mathrm{s}}\right)$ в $(6)$ определяется следующей цепочкой равенств:

$$
\begin{aligned}
P_{1}^{(1)} & =-c_{2}[1]-d_{2}[1]=-c_{3}[0,1]-d_{3}[0,1]=-c_{4}[0,0,1]-d_{4}[0,0,1]=\cdots= \\
& =-c_{n}[\underbrace{0,0, \ldots, 1}_{n-1}]-d_{n}[\underbrace{0,0, \ldots, 1}_{n-1}]=C_{\mathrm{F}}\left(-\frac{21}{8}+3 \zeta_{3}\right) .
\end{aligned}
$$

Второй член $P_{1}^{(2)}$ этого же полинома, аналитически фиксированный в $(15)$, также связывает различные элементы $\beta$-представлений:

$$
\begin{aligned}
P_{1}^{(2)} & =-c_{3}[1]-d_{3}[1]-d_{1}\left(c_{2}[1]-d_{2}[1]\right)= \\
& =-c_{4}[0,1]-d_{4}[0,1]-d_{1}\left(c_{3}[0,1]-d_{3}[0,1]\right)=\cdots= \\
& =-c_{n}[\underbrace{0, \ldots, 1}_{n-2}]-d_{n}[\underbrace{0, \ldots, 1}_{n-2}]-d_{1}\left(c_{n-1}[\underbrace{0, \ldots, 1}_{n-2}]-d_{n-1}[\underbrace{0, \ldots, 1}_{n-2}]\right)= \\
& =\left(\frac{397}{96}+\frac{17}{2} \zeta_{3}-15 \zeta_{5}\right) C_{\mathrm{F}}^{2} .
\end{aligned}
$$

Однако для получения аналитических значений коэффициентов $P_{1}^{(3)}$ и $P_{2}^{(2)}$ с применением метода $\beta$-разложения необходимо найти представления для коэффициентов четвертого порядка в рядах ТВ для функций $D\left(a_{\mathrm{s}}\right)$ и $C^{\mathrm{Bjp}}\left(a_{\mathrm{s}}\right)$.

Это может быть сделано после дополнительных аналитических вычислений вкладов глюино в эти важные физические величины в четвертом порядке ТВ и учете трехпетлевых глюонных эффектов в РГ $\beta$-функции КХД, вычисленных в схеме $\overline{\mathrm{MS}}$ в работе [50].

Полученные выше соотношения позволяют вывести новое теоретическое выражение для суммы коэффициентов четвертого порядка ТВ $d_{4}+c_{4}$. Для этого фиксируем число ароматов фермионов $n_{\mathrm{f}}$ из условия $\beta_{0}\left(n_{\mathrm{f}}=n_{0}\right)=0$, что соответствует анзацу Банкса-Закса [51] и приводит к значению $T_{\mathrm{F}} n_{0}=(11 / 4) C_{\mathrm{A}}$. В этом случае получаем

$$
\begin{aligned}
c_{4}\left(n_{0}\right)+d_{4}\left(n_{0}\right)= & c_{4}[0]+d_{4}[0]+\beta_{2}\left(n_{0}\right)\left(c_{4}[0,0,1]+d_{4}[0,0,1]\right)+ \\
& +\beta_{1}\left(n_{0}\right)\left(c_{4}[0,1]+d_{4}[0,1]\right) .
\end{aligned}
$$

Слагаемые в правой части формулы (32) известны из выражений $(21),(30)$ (а именно, $\left.-c_{4}[0,0,1]-d_{4}[0,0,1]\right)$ и из выражения $(31)$ (член $\left.-c_{4}[0,1]-d_{4}[0,1]\right)$. Подставляя в $\beta_{1}$ и $\beta_{2}$ фиксированное выше значение $n_{0}$, из соотношения (32) получаем

$$
\begin{aligned}
d_{4}\left(n_{0}\right) & +\mathrm{c}_{4}\left(n_{0}\right)=-\frac{333}{1024} C_{\mathrm{F}}^{4}+C_{\mathrm{A}} C_{\mathrm{F}}^{3}\left(-\frac{1661}{3072}+\frac{1309}{128} \zeta_{3}-\frac{165}{16} \zeta_{5}\right)+ \\
& +C_{\mathrm{A}}^{2} C_{\mathrm{F}}^{2}\left(-\frac{3337}{1536}+\frac{7}{2} \zeta_{3}-\frac{105}{16} \zeta_{5}\right)+C_{\mathrm{A}}^{3} C_{\mathrm{F}}\left(-\frac{28931}{12288}+\frac{1351}{512} \zeta_{3}\right) .
\end{aligned}
$$


Фиксируя число ароматов кварков $n_{\mathrm{f}}=n_{0}$ в конкретном аналитическом результате для $d_{4}\left(n_{0}\right)+c_{4}\left(n_{0}\right)$, следующем из вычислений [33], находим согласие с правой частью формулы (33).

Таким образом, применение выведенного в настоящей работе в четвертом порядке ТВ нового вида обобщенного соотношения Крютера (6), а также метода $\beta$-разложения работы [43] и анзаца Банкса-Закса [51] позволили получить дополнительный аргумент в пользу правильности результатов сложных и длительных аналитических компьютерных вычислений, проведенных группой сотрудников ИЯИ, Института теоретической физики частиц (Карлсруэ) и НИИЯФ МГУ [33]. Кроме того, отсутствие в правой части (33) трансцендентных членов, пропорциональных $\zeta_{7}$ и $\zeta_{3}^{2}$, при обращении в нуль коэффициента $\beta_{0}$ подтверждает сделанное в [33] наблюдение о пропорциональности подобных вкладов в определенные в схеме $\overline{\mathrm{MS}}$ коэффициенты $d_{4}$ и $c_{4}$ (см. результаты работы [33] и выражение (8)) первому коэффициенту РГ $\beta$-функции КХД.

Благодарности. Настоящая работа докладывалась на XVI Международном семинаре по физике высоких энергий "Quarks-2010" (6-12 июня 2010 г., Коломна) и на Международном семинаре "Структура адронов и КХД: от низких к высоким энергиям-2010" (5-9 июля 2010 г., Гатчина). Авторы выражают признательность организаторам этих семинаров за приглашение. Мы благодарны К. Г. Четыркину и Д. И. Казакову за полезные вопросы и обсуждения, Д. Броэдхарсту, А.Г. Грозину и О.В. Теряеву за интерес к нашей работе, С. Бродскому, Р. Крютеру и П. Минковскому за поддержку и комментарии по литературе после появления электронного препринта. Одному из нас (А.Л. Катаеву) также приятно отметить интерес и внимательное отношение к данной работе А. В. Гаркуши. Работа выполнена при финансовой поддержке РФФИ (грант № 11-01-00182).

\section{Список литературы}

[1] В. А. Матвеев, Р. М. Мурадян, А. Н. Тавхелидзе, ЭЧАЯ, 2:1 (1971), 5-32.

[2] O. Aharony, S. S. Gubser, J. M. Maldacena, H. Ooguri, Y. Oz, Phys. Rept., 323:3-4 (2000), 183-386, arXiv: hep-th/9905111.

[3] M. Baker, K. Johnson, Physica A, 96:1-2 (1979), 120-130.

[4] V. M. Braun, G. P. Korchemsky, D. Mueller, Prog. Part. Nucl. Phys., 51:2 (2003), 311-398, arXiv: hep-ph/0306057.

[5] R. J. Crewther, Phys. Rev. Lett., 28:21 (1972), 1421-1424.

[6] S. L. Adler, C. G. Callan, D. J. Gross, R. Jackiw, Phys. Rev. D, 6:10 (1972), 2982-2988.

[7] Н.Н. Боголюбов, Д. В. Ширков, Введение в теорию квантованных полей, Наука, М., 1984.

[8] M. S. Chanowitz, J. R. Ellis, Phys. Lett. B, 40:3 (1972), 397-400.

[9] N. K. Nielsen, Nucl. Phys. B, 120:2 (1977), 212-220.

[10] S. L. Adler, J. C. Collins, A. Duncan, Phys. Rev. D, 15:6 (1977), 1712-1721.

[11] J. C. Collins, A. Duncan, S. D. Joglekar, Phys. Rev. D, 16:2 (1977), 438-449.

[12] P. Minkowski, On the anomalous divergence of the dilatation current in gauge theories, Preprint № 76-0813, Univ. of Bern, Bern, 1976.

[13] D. J. Broadhurst, A. L. Kataev, Phys. Lett. B, 315:1-2 (1993), 179-187, arXiv: hep-ph/9308274.

[14] S. G. Gorishny, A. L. Kataev, S. A. Larin, Phys. Lett. B, 259:1-2 (1991), 144-150.

[15] L. R. Surguladze, M. A. Samuel, Phys. Rev. Lett., 66:5 (1991), 560-563; Erratum, 66:18 (1991), 2416. 
[16] S. A. Larin, J. A. M. Vermaseren, Phys. Lett. B, 259:3 (1991), 345-352.

[17] K. G. Chetyrkin, Phys. Lett. B, 391 (1997), 402-412, arXiv: hep-ph/9608480.

[18] K. G. Chetyrkin, A. L. Kataev, F. V. Tkachov, Phys. Lett. B, 85:2-3 (1979), 277-279.

[19] M. Dine, J. R. Sapirstein, Phys. Rev. Lett., 43:10 (1979), 668-671.

[20] W. Celmaster, R. J. Gonsalves, Phys. Rev. Lett., 44:9 (1980), 560-564.

[21] S. G. Gorishny, S. A. Larin, Phys. Lett. B, 172:1 (1986), 109-112.

[22] E. B. Zijlstra, W. L. van Neerven, Phys. Lett. B, 297:3-4 (1992), 377-384.

[23] Г. Т. Габададзе, А. Л. Катаев, Писъма в ЖЭТФ, 61:6 (1995), 439-443.

[24] A.L. Kataev, "The generalized Crewther relation: the peculiar aspects of the analytical perturbative QCD calculations", Proceedings of the 2 nd Workshop on Continuous Advances in QCD (Minneapolis, MN, 28-31 March, 1996), ed. M. I. Polikarpov, World Scientific, Singapore, 1996, 107-132, arXiv: hep-ph/9607426, Preprint INR-0926-96.

[25] S. L. Adler, W. A. Bardeen, Phys. Rev., 182:5 (1969), 1517-1536.

[26] R. J. Crewther, Phys. Lett. B, 397:1-2 (1997), 137-142, arXiv: hep-ph/9701321.

[27] D. Muller, Private communication, 1996.

[28] S. J. Brodsky, G. T. Gabadadze, A. L. Kataev, H. J. Lu, Phys. Lett. B, 372:1-2 (1996), 133-140, arXiv: hep-ph/9512367.

[29] J. Rathsman, Phys. Rev. D, 54:5 (1996), 3420-3426, arXiv: hep-ph/9605401.

[30] G. Grunberg, A. L. Kataev, Phys. Lett. B, 279:3-4 (1992), 352-358.

[31] S. J. Brodsky, G. P. Lepage, P. B. Mackenzie, Phys. Rev. D, 28:1 (1983), 228-235.

[32] S. J. Brodsky, H. J. Lu, Phys. Rev. D, 51:7 (1995), 3652-3668, arXiv: hep-ph/9405218.

[33] P. A. Baikov, K. G. Chetyrkin, J. H. Kuhn, Phys. Rev. Lett., 104:13 (2010), 132004, 4 pp., arXiv: 1001.3606.

[34] O. V. Tarasov, A. A. Vladimirov, A. Y. Zharkov, Phys. Lett. B, 93:4 (1980), 429-432.

[35] S. A. Larin, J. A. M. Vermaseren, Phys. Lett. B, 303:3-4 (1993), 334-336, arXiv: hep-ph/9302208.

[36] P. A. Baikov, K. G. Chetyrkin, J.H. Kühn, "Massless propagators: applications in QCD and QED", Proceedings of 8th International Symposium on Radiative Corrections (RADCOR 2007): Application of Quantum Field Theory to Phenomenology (Florence, Italy, 1-6 October 2007), 2007, 023, arXiv: 0810.4048.

[37] A. L. Kataev, Phys. Lett. B, 668:4 (2008), 350-352, arXiv: 0808.3121.

[38] A. L. Kataev, Phys. Lett. B, 691:2 (2010), 82-86, arXiv: 1005.2058.

[39] A. L. Kataev, S. V. Mikhailov, "New extended Crewther-type relation", Proceeding of 9th International Symposium on Radiative Corrections (RADCOR 2009): Application of Quantum Field Theory to Phenomenology (Ascona, Switzerland, October 25-30, 2009), 2010, 036, arXiv: 1001.0728 .

[40] P. A. Baikov, K. G. Chetyrkin, J. H. Kühn, Phys. Rev. Lett., 101:1 (2008), 012002, 4 pp., arXiv: 0801.1821.

[41] T. van Ritbergen, J. A. M. Vermaseren, S. A. Larin, Phys. Lett. B, 400:3-4 (1997), 379-384, arXiv: hep-ph/9701390.

[42] M. Czakon, Nucl. Phys. B, 710:1-2 (2005), 485-498, arXiv: hep-ph/0411261.

[43] S.V. Mikhailov, JHEP, 06 (2007), 009, 28 pp., arXiv: hep-ph/0411397; "Any order generalization of BLM procedure in QCD", Proceedings of the 13th International Seminar Quarks 2004 (Pushkinskie Gory, Russia, May 24-30, 2004), 2, eds. D. G. Levkov, V. A. Matveev, V. A. Rubakov, INR, Moscow, 2005, 536-550.

[44] А. Л. Катаев, А. А. Пивоваров, Писъма в ЖЖЭТФ, 38:6 (1983), 309-310.

[45] L. J. Clavelli, L. R. Surguladze, Phys. Rev. Lett., 78:9 (1997), 1632-1635, arXiv: hep-ph/9610493.

[46] D. I. Kazakov, Nucl. Phys. Proc. Suppl., 203-204 (2010), 118-154, arXiv: 1010.5419. 
[47] S. Akula, N. Chen, D. Feldman, M. Liu, Z. Liu, P. Nath, G. Peim, Phys. Lett. B, 699:5 (2011), 377-382, arXiv: 1103.1197.

[48] M. E. Machacek, M. T. Vaughn, Nucl. Phys. B, 236:1 (1984), 221-232.

[49] C. N. Lovett-Turner, C. J. Maxwell, Nucl. Phys. B, 452:1-2 (1995), 188-212, arXiv: hep-ph/9505224.

[50] L. Clavelli, P. W. Coulter, L. R. Surguladze, Phys. Rev. D, 55:7 (1997), 4268-4272, arXiv: hep-ph/9611355.

[51] T. Banks, A. Zaks, Nucl. Phys. B, 196:2 (1982), 189-204. 